\title{
Campeonatos deportivos y bolsa, un acercamiento a la dinámica del mercado
}

\author{
Iván Francisco Tunjano Pinzón ${ }^{28}$
}

\section{Introducción}

4 l entorno competitivo de las actividades deportivas como fútbol, tenis, béisbol, baloncesto, ciclismo, voleibol, etc., en las que se crean equipos de jugadores que luego se enfrentan entre sí en un campeonato o modelo de eliminación que busca resaltar sus cualidades deportivas, se puede comparar con el mundo empresarial en el que un escenario de competencia puede llegar a definirse de acuerdo con las diferencias comparativas entre las empresas, que llevan a que algunas sobresalgan y, por consiguiente, adquieran más utilidades sobre sus inversiones; incluso en un mismo sector y un mismo espacio de mercado, algunas empresas logran generar mayores beneficios. En línea con esto, puede tener mucho sentido comparar un mundo empresarial con un escenario deportivo de competencias.

Del lado de los inversionistas, podríamos mencionar que se parecen mucho a los seguidores o hinchas de un equipo en cualquier deporte: los fanáticos de un equipo normalmente están enterados sobre las decisiones que toman sus directivos y sobre las estrategias que se plantean en el terreno de juego. De la misma forma, los inversionistas se enteran de la compra de acciones de una empresa, revisan sus estados financieros y conocen cualquiera de los comunicados que realice la junta directiva y sus directivos. La principal diferencia entre estas comparaciones radica en que un inversionista, cuando no

28. Docente Auxiliar de Finanzas. Institución Universitaria Politécnico Grancolombiano. Ingeniero Industrial, Especialista en Finanzas y Máster en Asesoramiento Financiero. 
se siente cómodo con la estrategia propuesta o considera que no es acertada, simplemente "cambia de equipo", vende las acciones de la empresa y compra nuevas acciones en otra compañía; entretanto, los hinchas permanecen a largo plazo siendo parte de los equipos que han escogido. Sin este último atributo sería factible comparar de forma directa los inversionistas como hinchas o fanáticos de un equipo, y el equipo en sí con una empresa.

Profundizando más en esta idea, podemos ver que en el ámbito deportivo algunos equipos o clubes compran y venden jugadores, esto es, se ha creado un mercado que funciona con principios económicos (Szymanski, 2003) ¿Por qué hacen este ejercicio? Se trata de un ejercicio netamente financiero en el que inicialmente un club deportivo puede contratar un jugador y adquirir sus derechos (solo si ningún club los ha adquirido previamente), y posteriormente, teniendo en cuenta el desempeño del jugador, estos derechos pueden ser transados con otros clubes que puedan tener mayores recursos económicos o pertenecer a ligas superiores, dando así una ganancia para el club poseedor de los derechos iniciales.

Podemos decir, entonces, que los jugadores son como títulos valores, que ganan o pierden valor de acuerdo con su desempeño (tengamos en cuenta que los jugadores son seres humanos, por lo que este ejemplo no busca mostrarlos como "cosas"). Continuando con nuestra idea, los jugadores y su desempeño se pueden comparar con un título valor negociable, guardando las proporciones, ya que el primero es un ser humano y el segundo es un activo.

En síntesis, los campeonatos deportivos entre equipos se parecen a lo que ocurre en un mercado de valores, donde los equipos corresponden a las empresas, y los inversionistas se parecen a sus respectivos hinchas, teniendo presente que su única diferencia es que los inversionistas se cambian de "equipo", es decir, de empresa, de acuerdo con sus propias necesidades de rentabilidad, y no crean apegos como ocurre en el caso de los fanáticos.

\section{Metodología}

En el área de Finanzas de la Institución Universitaria Politécnico Grancolombiano se realizó una actividad asociando los escenarios previamente descritos, como estrategia de enseñanza sobre el funcionamiento del mercado de valores. El uso de deportes como estrategia didáctica ha probado ser efectiva para despertar el interés en temas financieros, como lo documentan Mahar y Rodney (2010). La referida actividad se desarrolló en el mes de junio de 2018 y se denominó "subasta de equipos y campeonato deportivo para inversionistas". En dicha actividad se desarrolló un campeonato de fútbol en PlayStation y Xbox, mediante el cual los estudiantes de carreras de Administración y Negocios internacionales tomaron dos tipos de roles, a saber: 
- Un grupo de ocho estudiantes asumieron el rol jugador, que para este caso se puede comparar a los CEO de una empresa, y jugaron entre sí en un campeonato con la estructura descrita en la ilustración 3.

- Otro grupo de estudiantes, sin límite de participantes, asumieron el rol de inversionistas (fanáticos o hinchas de los equipos).

- Un grupo de profesores tomaron el rol de proveedores de infraestructura, es decir, la bolsa de valores y el depósito central de valores (ver los roles en la ilustración 3).

Ilustración 3. Roles asignados para la realización del juego

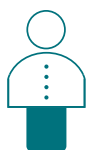

Proveedor de infraestructura

BVC

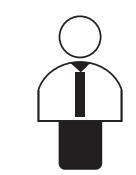

Inversionista

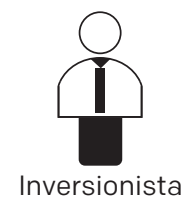

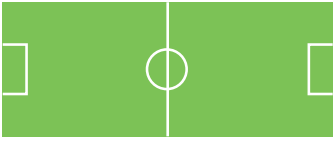

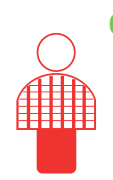

Jugador

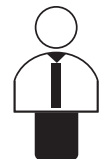

Inversionista
Consola

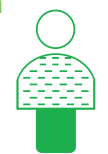

Jugador
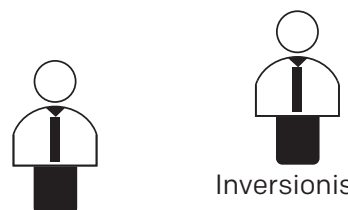

Inversionista

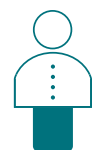

Proveedor de infraestructura

Depósito de

Valores

Inversionista

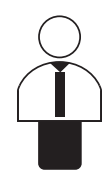

Inversionista

Fuente: elaboración propia (2018).

Ilustración 4. Estructura del torneo y Cuadro de eliminación

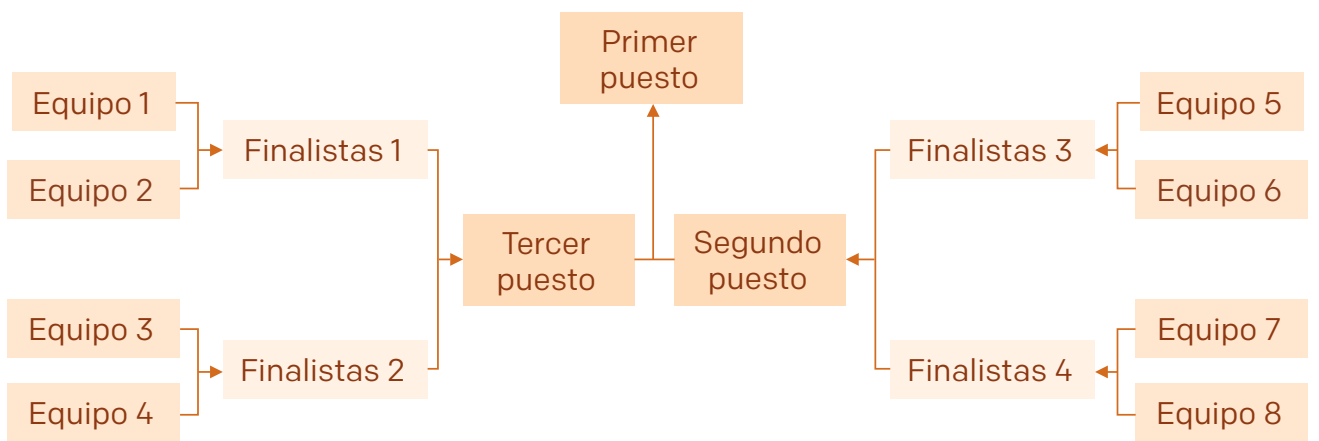

Fuente: elaboración propia (2018). 
Para desarrollar este escenario de inversión, se buscó hacer que los estudiantes aprendieran mediante una estrategia de gamificación de un proceso de inversión, siguiendo los estándares propuestos por Israel (2017), y por esto se vinculó al proceso una consola de videojuego que puede ser PlayStation $\AA$ o Xbox $\AA$, donde de manera simultánea se pueden enfrentar los diferentes estudiantes que tienen el rol de CEO, para buscar llegar a la mejor posición posible en el cuadro de eliminación presentado en la ilustración 4.

De manera previa al inicio del campeonato, se socializó un modelo de educación basado en algunas prácticas tradicionales que realizan los intermediarios de valores, como las sociedades comisionistas de bolsa en vísperas al mundial de fútbol, en el que mediante una subasta se venden de forma simbólica los equipos que participan de este campeonato mundial en una rueda de negociación abierta en la que cualquiera puede llegar a ofrecer y comprar el equipo (físicamente se elaboran banderas que luego son entregadas a los compradores, simbolizando la compra del equipo). El juego consiste en asociar las banderas con ACCIONES, es decir, con títulos de participación que pueden llegar a ser comprados por varios participantes o un participante, en adelante INVERSIONISTAS, como se muestra en la ilustración 5.

El objetivo de la compra o la inversión es lograr ganar dinero adicional al final del campeonato que se juega en la vida real, y del cual no se conocen los resultados; sin embargo, se tiene alguna información por los procesos de clasificación y por el desempeño de los equipos, así como por el estilo de dirección y estrategias que se conozcan de cada uno de los directores técnicos, que en este caso los podríamos asemejar a los CEO de las empresas.

Ilustración 5. Proceso de subasta
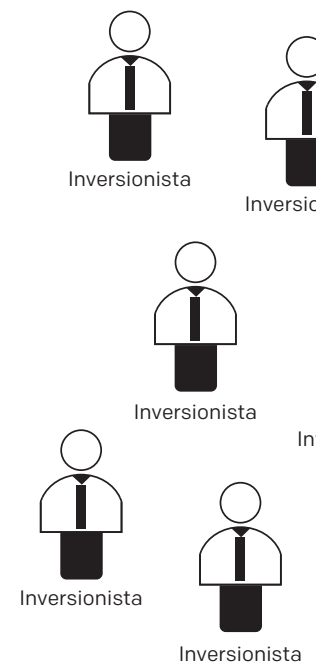
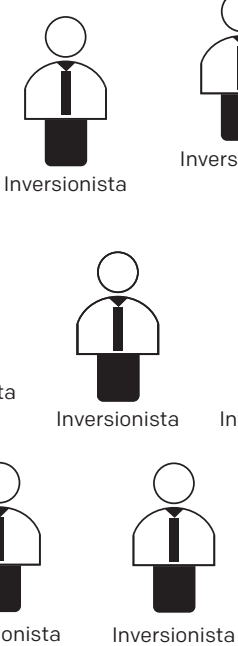
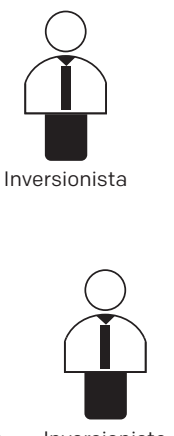

nversionista
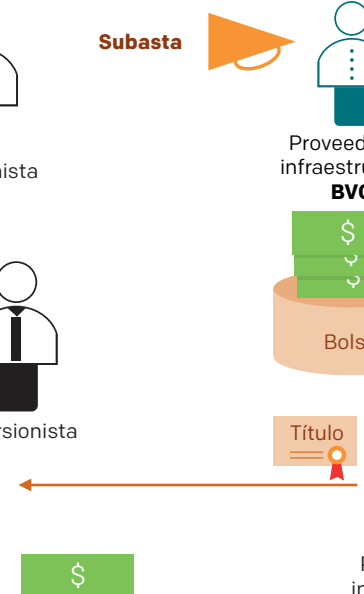

$$
\begin{aligned}
& \text { infraestruc } \\
& \text { BVC }
\end{aligned}
$$
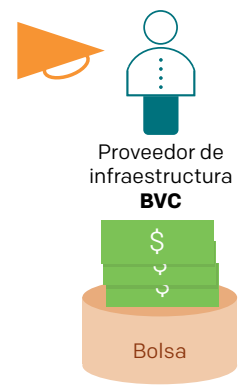

Equipo 2

Equipo 1
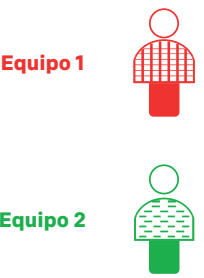

Equipo 3

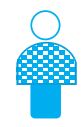

Equipo 4

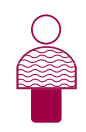

Fuente: elaboración propia (2018). 
Finalmente, como ocurre en la vida real de un mercado de valores, cuando un inversionista compra acciones de una empresa no compra el pasado ni compra el presente, sino que compra el futuro que puede llegar a tener la empresa, es decir, su expectativa de desempeño, la cual, en la medida que va corriendo el tiempo, puede dar ganancias o pérdidas.

De esta forma, en esta subasta de equipos, el valor futuro que permitirá a los inversionistas calcular su rentabilidad dependerá de la posición que ocupen dentro del campeonato, teniendo presente que se asignarán porcentajes para los primeros cuatro puestos y para los últimos tres puestos: cada posición gana un porcentaje del valor total que se reúna en la subasta, y esto ocurre una vez termina el campeonato.

Es decir, si el primer puesto tiene como premio el 20\%, entonces se ganaría el $20 \%$ del total del dinero que se reúna en la subasta de los 32 equipos de un mundial de fútbol, como se muestra en la ilustración 6.

Para el ejercicio realizado en el aula de clases, se asignó la siguiente tabla de posiciones, el valor futuro de las banderas o equipos que, después de realizado dicho campeonato, ocuparán de acuerdo con el cronograma de eliminación mencionado anteriormente:

Ilustración 6. Cuadro de premiación

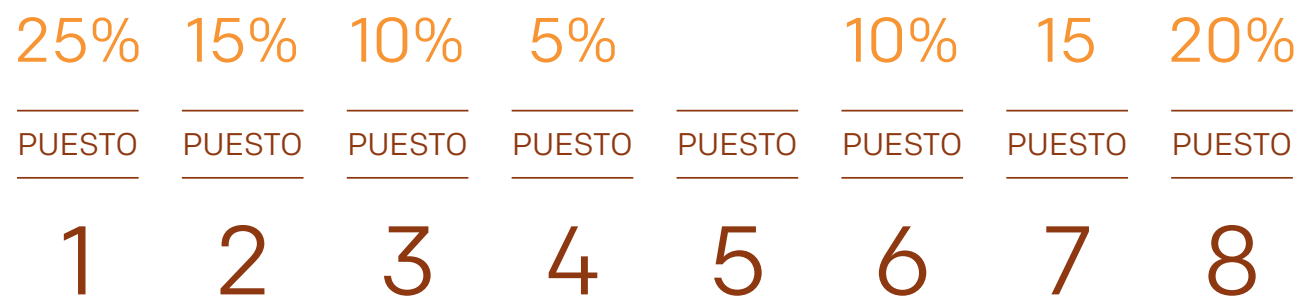

Fuente: elaboración propia (2018).

\section{¿Cómo lograr que la inversión genere rendimientos?}

Estas inversiones generan rendimientos de acuerdo con la comparación entre el precio de compra (valor subastado por el equipo comprado), y la expectativa de la posición que ocupe.

En este sentido, suponiendo que un equipo sea subastado en $\$ 150.000$ y el valor de la subasta total sume $\$ 2.900 .000$, y esperando que ocupe el primer lugar, entonces el "Inversionista" podrá esperar un retorno de $\$ 725.000$, es decir $383.33 \%$ de rentabilidad. Pero si, por el contrario, la posición que ocupa este equipo en la tabla de posiciones es la cuarta, 
entonces el “Inversionista” podrá esperar un retorno de \$145.000, y en este caso su rentabilidad sería negativa de $-3.33 \%$.

Estas rentabilidades serían posibles si no existiera el mercado secundario, el cual corresponde al mercado creado por "inversionistas" con "inversionistas", que intercambian sus activos de acuerdo con sus expectativas de precio en el futuro, y teniendo en cuenta que durante un partido de clasificación "todo puede pasar" (que se clasifique un equipo en último minuto, que el equipo con mejor juego pierda, que el equipo perdedor gane en último minuto, que se cobre un penalti inesperado, etc.), entonces la ganancia no está asegurada hasta tanto no se termine el juego, lo que les da la oportunidad a los "inversionistas" de realizar ventas parciales o totales de su equipo, y de esta manera jugar un poco con el valor futuro que pueda tener su inversión. En este sentido, podríamos tener el escenario negativo mencionado en el párrafo anterior, sin embargo, el "inversionista" inicial durante alguno de los partidos jugados pudo haber vendido el 50\% de participación en un precio de 90.000, generando para su inversión el siguiente resultado integrado:

Ilustración 7. Cuadro resumen de un inversionista

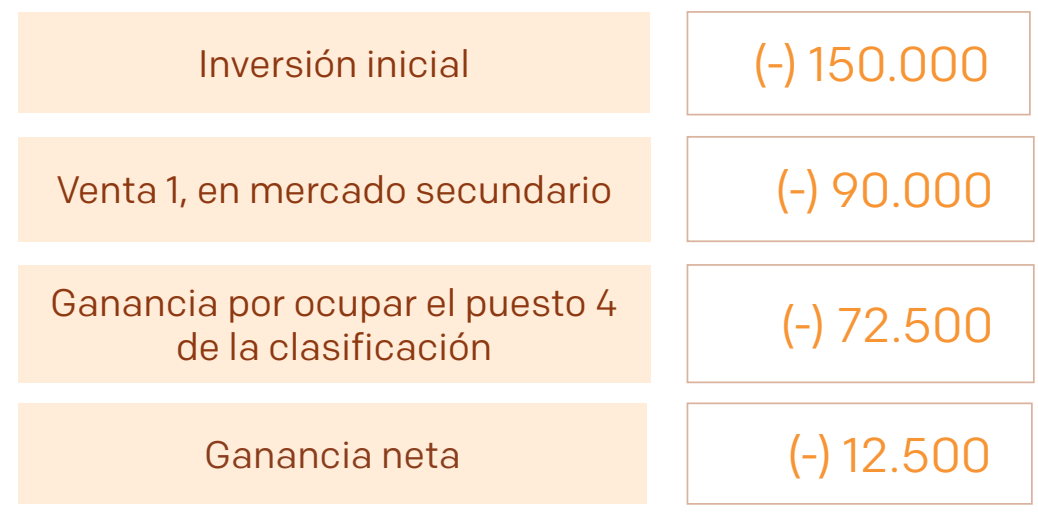

Fuente: elaboración propia (2018).

Como se puede observar en este ejemplo, el inversionista inicial logra tener una rentabilidad positiva de $8.33 \%$ sobre una inversión que inicialmente habría generado una rentabilidad negativa de $-3.33 \%$. Esta situación permite establecer un paralelo con el mercado de valores real, donde diferentes estrategias de inversión, incluso con activos que no generen al final una valorización positiva, resultan en rendimientos positivos para los inversionistas que ejecutan diferentes estrategias para conseguir mejores rentabilidades, incluso saliendo a vender activos a pérdida para tomar utilidades posteriormente en otros activos de inversión. 


\section{El mercado primario en esta actividad}

Un mercado primario se define como el escenario donde un emisor de valores realiza la venta inicial de un título valor de renta fija o de renta variable, es decir, la emisión inicial. En este mercado existen diferentes metodologías de emisión, como ventas directas, o ventas abiertas a todo tipo de inversionistas, subastas (en diferentes modalidades), emisiones con comisión de colocación, colocaciones en firme, entre otras.

Para el ejercicio descrito en este artículo, la modalidad escogida es una subasta abierta con posibilidad de pujar por el precio, que significa una mejora constante del precio de compra, y la asignación de título valor "banderas simbólicas de cada equipo" se hace al mejor postor en esta subasta.

En este sentido es muy importante que los participantes aprecien y den un seguimiento al valor de subasta de cada equipo, por cuanto de este valor dependerá el posible valor futuro que se obtenga de sus inversiones y, en consecuencia, en el desarrollo de la subasta los ánimos pueden mejorar al proyectar mayores valores de compra (Inversión), derivados de una expectativa de valor futuro superior.

Ilustración 8: Asignación del valor total de la subasta

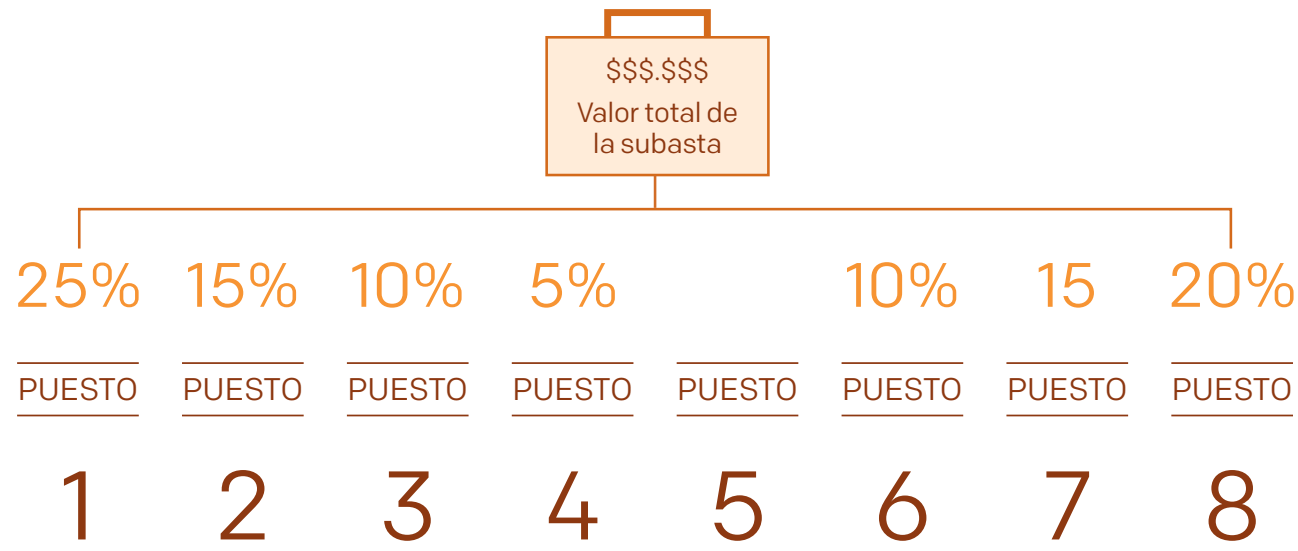

Fuente: elaboración propia (2018).

Por ejemplo, si el valor de la subasta se encuentra en $\$ 1.900 .000$ y se está subastando un equipo del cual se tienen expectativas altas y se espera que ocupe el primer puesto, entonces el mayor valor que podría alcanzar sería el 25\% del valor actual de la subasta, es decir, \$475.000. Sin embargo, este valor podría ser superior en la medida que falten equipos por subastar que puedan llevar el valor total de la subasta a nuevos valores por encima de $\$ 1.900 .000$. 


\section{Como desarrollar el mercado secundario}

El mercado secundario está definido como un escenario donde inversionistas intercambian títulos con otros inversionistas, mediante un sistema de negociación que puede ser electrónico y automatizado, y en el que los inversionistas colocan sus posturas de compra y venta y estas se calzan de forma automática, o mediante un sistema de registro donde ser ingresan las operaciones realizadas en el mercado OTC (over the counter). Para este ejercicio, las operaciones se registrarían de forma OTC, por lo que cada negociación termina siendo pactada entre los participantes y por los participantes "inversionistas".

En el mercado secundario existe una oportunidad muy importante para mejorar la inversión realizada en el mercado primario, ya que, dependiendo del desarrollo del campeonato y desempeño de cada equipo en cada partido, se pueden realizar operaciones de compra y venta de participaciones en cada activo de inversión "Equipos".

Este es el escenario más propicio para que cada estudiante que participe de esta actividad logre vincular sus competencias propias en negociación con estrategias y con operaciones de intercambio de activos que les permitan acrecentar el valor de su inversión, sin que el concurso se haya terminado por completo. De esta forma, un participante puede llegar a generar mejores rendimientos que incluso habiendo comprado desde el inicio el equipo que ocupe el primer puesto.

\section{Algunos aspectos a tener en cuenta}

Organizadores:

- La tabla de premiación se puede alterar asignando del 100\% algunos premios a los equipos que logren las menores diferencias de gol, o goles realizados por defensas, etc. Sin embargo, es necesario premiar siempre las últimas posiciones del campeonato.

- En la subasta se deben poner reglas como la oferta mínima aceptada, ejemplo, de 100 en 100; de esta forma, ningún proponente puede hacer ofertas de 110 o 111.

- La subasta debe ser dinámica y debe buscar acrecentar el valor de la bolsa general.

- Bolsa de valores:

- Se debe establecer una bolsa de valores (puede ser una persona), que registre el valor de las ventas y realice la compensación de las operaciones.

- Durante el campeonato, cada una de las operaciones realizadas en el mercado secundario debe ser registrada por la bolsa de valores y allí se conservan los registros de cada negocio realizado.

- La bolsa puede establecer el cobro de comisiones proporcionales a los valores negociados para ofrecer una experiencia más real del proceso. Esta comisión no puede llegar a limitar la realización de negocios, por ser muy alta. 


\section{Conclusiones y recomendaciones}

El uso de un torneo jugado con consolas como Xbox o PlayStation permite poner a prueba las habilidades de negociación y de creación de estrategias de inversión de los estudiantes que cumplen el rol de inversionistas. Igualmente, permite explicarles el rol que tienen algunos intermediarios de valores como la Bolsa de Valores, que desarrolla y administra el sistema de negociación; los depósitos centralizados de valores, que permiten que los títulos valores se negocien de forma desmaterializada, evitando diferentes problemas que se derivan de la negociación física de títulos valores.

Por parte de los estudiantes que desarrollan el rol de CEO, es decir, que comandan un equipo en el proceso de eliminación, también generan una experiencia constructiva en la medida que se responsabilizan por la generación de ganancias para cada uno de los inversionistas que confían en el buen desempeño que tendrán durante los juegos que disputen.

Sin duda la experiencia y el desarrollo de nuevas habilidades de inversión y de conformación de estrategias es uno de los mayores beneficios que puede tener esta actividad para los estudiantes.

El proceso de subasta debe estar acompañado de algún elemento sonoro que genere persuasión en los participantes y los ayude a animarse: ecos de barras, música relacionada con actividades deportivas, entre otros. Al mismo tiempo es importante que se lleven al aula de clases elementos decorativos alusivos a los equipos que se van a subastar, con el ánimo de ambientar el escenario.

Durante el proceso de subasta es importante que se hable previamente de la experiencia de juego de los CEO, es decir, de los estudiantes que cumplen dicho rol, con el objetivo de que los inversionistas tengan información fundamental para tomar decisiones de inversión.

El docente que realice la subasta debe imprimir emoción mediante comentarios que generen motivación en los estudiantes que cumplen el rol de inversionista. Esto se hace con el fin de que la bolsa de dinero didáctico crezca lo mayor posible.

Es importante precisar que realizar esta actividad requiere de un conocimiento profundo del funcionamiento del mercado público de valores, por eso es importante, en lo posible, sumar profesionales con experiencia en administración de portafolios que puedan motivar a los estudiantes que manejan el rol de inversionistas a realizar diferentes operaciones de compra, venta, REPOS e incluso opciones CALL y PUT durante el desarrollo del campeonato, para de esta forma lograr en el estudiante la experiencia de administrar un portafolio, en este caso de equipos, y lograr que su beneficio económico crezca durante todo el proceso, así como se les enseña de esta forma a tolerar la frustración generada por una mala inversión y administrar el riesgo asociado a las mismas.

Durante el proceso de juego es importante que los docentes puedan proponer a los 
estudiantes estrategias que les ayuden a recuperar sus portafolios o que les permitan asegurar rendimientos obtenidos, es decir, cerrar los riesgos de inversiones que les puedan estar dando frutos durante el desarrollo del juego.

El proceso de subasta se debe hacer con dinero didáctico, porque es un ejercicio académico y no de inversión real.

\section{Referencias}

Israel, M. (2017). Game-based learning and gamification. Recuperado de: http://micheleisrael.com/wp-content/uploads/2017/11/Game-Based-Learning-Gamification.pdf

Mahar, J., y Rodney, P. (2010). Using sports to teach finance and economics. Journal of economics and finance education, 9(2), 43-53.

Szymanski, S. (2003). The Assessment: The economics of sports. Oxford Review of Economic Policy, 19(4), 467-477. 